\title{
CT Image Segmentation Algorithm Based on Merging of Similar Adjacent Components
}

\author{
Xueguang Sun and Weigen Qiu \\ Guangdong University of Technology, Guangzhou, 510006, Guangdong, China
}

\begin{abstract}
CT is one of the most widely used medical imaging techniques. Image segmentation is of great significance to medical image aided clinical diagnosis. Due to the complexity of structure and shape of CT image, and the inhomogeneity of organization, the commonly used image segmentation algorithm can not meet the requirements. Therefore, a CT image segmentation algorithm based on similarity criterion of adjacent components is proposed in this paper. The component tree is built quickly for the CT image, and the component tree protocol is carried out according to the attribute similarity criterion of adjacent components to complete the segmentation target. The experimental results show that the segmentation results are more accurate and more efficient.
\end{abstract}

Keywords-CT images; adjacent components; image segmentation

\section{INTRODUCTION}

Image segmentation is to separate the subregion of the target image from the complex background, and it is the basis of the higher level image analysis. In recent years, scholars have proposed a large number of excellent segmentation algorithms[1]. These algorithms can be roughly divided into the following: voxel based image segmentation, region based image segmentation, local priori based image segmentation, image segmentation based on global character priori and artificial intelligence based segmentation. [2,3] Medical image segmentation has been a hot research field in medical image processing. Frank et al. [4] successfully segmented the gray matter, white matter and cerebrospinal fluid fluid in the T1 weighted brain MR images by using the optimal threshold. In document [5], Wang $\mathrm{H}$ and so on use region based segmentation method to synthesize pixel information and local relationship between pixels, and have been applied to hippocampus segmentation successfully. The Snake method [6] is based on the priori of the local shape, and is suitable for the segmentation of various shape targets, and is widely used in two-dimensional image segmentation.

Compared with ordinary image segmentation, medical image segmentation has higher requirements for the precision of image segmentation and the speed of segmentation because of its special domain. In this paper, the CT image segmentation method based on component tree is studied. In this paper, we first use the algorithm of [7] to set up the component tree of the image, and insert the gray and area dual attributes in its component nodes. Then we merge the similar components with the adjacent components' gray similarity decision rule to complete the segmentation of CT images. Finally, the experimental results show that the segmentation method has low time complexity and high algorithm efficiency, which not only can effectively remove the noise in the image, but also suppress the over segmentation phenomenon, and achieves the desired segmentation effect. The algorithm flow of this article is shown in Figure I.

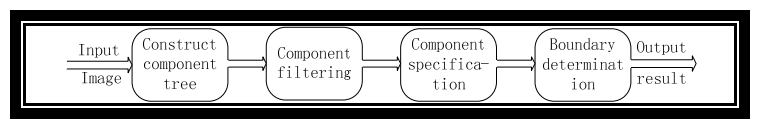

FIGURE I. THE FLOW CHART OF THE ALGORITHM IN THIS PAPER

\section{BASIC NOTIONS}

Let $\Omega$ is a nonempty finite set, $\mathrm{V}$ is a non empty finite poset, which is supposed to have the minimum value of $\perp$. An image is the function $\mathrm{f}: \Omega-\mathrm{V}$, the set $\Omega$ and the $\mathrm{V}$ are called the support set and the value space of the image $f$, respectively.

The following is a part of the definition about the concept of the component tree.

Definition 1: Let $\mathrm{X} \subseteq \Omega$ and $\mathrm{v} \in \mathrm{V}$, threshold function $\lambda \mathrm{v}$ : $\mathrm{V} \Omega \rightarrow 2 \Omega$, for any $\mathrm{f}: \Omega \rightarrow \mathrm{V}, \lambda \mathrm{v}(\mathrm{f})=\{\mathrm{x} \in \Omega \mid \mathrm{f}(\mathrm{x}) \geq \mathrm{v}\}$. for any $\mathrm{v} \in \mathrm{V}$, all of the Connected branches in the threshold set $\lambda \mathrm{v}(\mathrm{f})$ noted $\mathrm{C}[\lambda \mathrm{v}(\mathrm{f})]$.

Definition 2: Let $\mathrm{X} \subseteq \Omega$ and $\mathrm{v} \in \mathrm{V}$, barrel function $\mathrm{C}(\mathrm{X}, \mathrm{v})$ : $\Omega \rightarrow \mathrm{V}$, for any $\mathrm{x} \in \mathrm{X}, \mathrm{C}(\mathrm{X}, \mathrm{v})(\mathrm{x})=\mathrm{v}$; or else $\mathrm{C}(\mathrm{X}, \mathrm{v})(\mathrm{x})=\perp$. Using the threshold function $\lambda \mathrm{v}$, the $\mathrm{f}$ can be decomposed into some bucket functions. On the contrary, the image $f$ can also be reconstructed by these bucket functions, $f=\vee_{v} \in V_{V} \vee_{X} \in C[\lambda(f)]$ $\mathrm{C}(\mathrm{X}, \mathrm{v}) \vee$ notes that supremum function of the points set function. The Connected branch $\mathrm{X}$ may belong to more than one $\mathrm{C}[\lambda \mathrm{v}(\mathrm{f})]$, noted that $\mathrm{m}(\mathrm{X})=\max \{\mathrm{v} \in \mathrm{V} \mid \mathrm{X} \in \mathrm{C}[\lambda \mathrm{v}(\mathrm{f})]\}$, generally, $\mathrm{m}(\mathrm{X})=\min \mathrm{x} \in \mathrm{X}\{\mathrm{f}(\mathrm{x})\}$.

We note that $\Psi=\{(\mathrm{X}, \mathrm{m}(\mathrm{X})) \mid \mathrm{X} \in \mathrm{Uv} \in \mathrm{VC}[\lambda \mathrm{v}(\mathrm{f})]\}$, The definition of partial order relation in $\Psi$ : $(\mathrm{X} 1, \mathrm{~m}(\mathrm{X} 1)) \leq(\mathrm{X} 2$, $\mathrm{m}(\mathrm{X} 2)) \Leftrightarrow \mathrm{X} 1 \subseteq \mathrm{X} 2$. As shown in Figure II, a schematic diagram of the image f component tree is constructed.

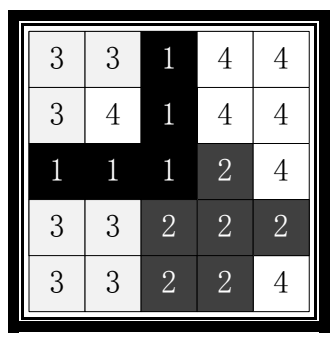

(A) $F$ 


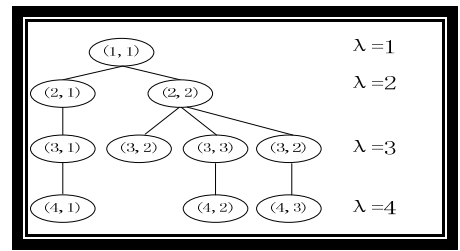

(B) COMPONENT TREE OF $F$

FIGURE II. (A) THE IMAGE $F$ AND THE COMPONENT TREE OF $F$.

\section{Algorithm of Merging of Similar Adjacent COMPONENTS}

L.Najman and M.courprie, Bertrend and others propose to use component tree structure to represent images, which lays a deep theoretical foundation for the establishment of component tree. Generally, in constructing the component tree, every node has qualitative or quantitative statistical information such as shape, area and so on. This information provides a basis for further image manipulation, such as the pruning of a tree, that is, the so-called image filtering. Set pruning function Fp, which is based on a $\mathrm{p}$ test predicates on the attribute set. This pruning function can remove the nodes that does not meet the test of the $\mathrm{P}$, the pixel reduction to other nodes nearby, so the triplet $(\Psi, \mathrm{L}$, R) called component tree of $\mathrm{f}$ evolved into ( $\Psi^{\prime}, \mathrm{L}$ ', R), note that $\mathrm{Fp}(\Psi, \mathrm{L}, \mathrm{R})=\left(\Psi^{\prime}, \mathrm{L}^{\prime}, \mathrm{R}\right)$. This process is called the reduction of the component tree.

This algorithm includes four processes, such as image preprocessing, image component tree construction, component specification and boundary determination.

\section{A. Image Preprocessing}

The image preprocessing method adopted in this paper is Gauss Laplasse (LoG) transformation. Experimental results are shown in Figure 4. After LoG transformation, the image noise is effectively suppressed, and the image details are significantly enhanced. The Gauss Laplasse operator is:

$$
\operatorname{LoG}=\frac{x^{2}+y^{2}-2 \sigma^{2}}{\sigma^{4}} \mathrm{e}^{-\left(x^{2}+y^{2}\right)} / 2 \sigma^{2}
$$

The smaller the sigma, the better the enhancement, and on the contrary, the better the smoothness. The proper Sigma value should be selected during the enhancement process. The value of the sigma in the experiment, shown in Figure IV, is 0.8 .

\section{B. The Construction of Component Tree}

We use the immersion algorithm to construct the component tree quickly. In the process of constructing the component tree, using find-union algorithm aimed to implement path compression. As shown in Figure III (A), a simple graph $\mathrm{f}$ is a four connection, and all the pixels of the image $f$ are the full order relation on the $\mathrm{R}$, and are descended in descending order by the gray value.

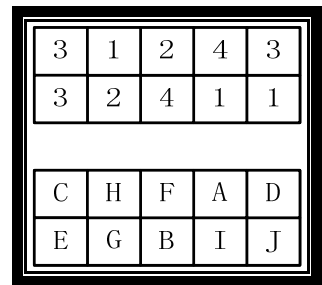

(A) SAMPLE IMAGE F (TOP) AND THE ORDERING BETWEEN PIXELS (BUTTOM)

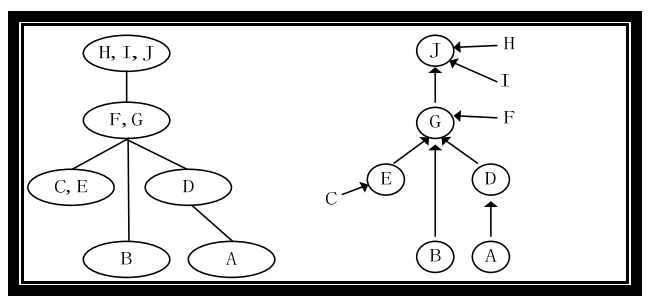

(B) COMPONENT TREE OF $F$

(C) COMPONENT TREE OF $F$

BEFORE PATH COMPRESSION

AFTER PATH COMPRESSION

\section{FIGURE III COMPONENT TREE CONSTRUCTION PROCESS}

\section{Component Reduction and Boundary Determination}

The first constructed component tree contains a large number of similar constructs. The component reduction should be based on two basic principles: (1) the gray difference between the two components; (2) the difference between the pixels in the component.

1) The calculation of the node area;

2) Find the gray value of the node;

3) component tree reduction;

The area change rate between two components $\mathrm{C} 1$ and $\mathrm{C} 2$ is defined, and the rate of gray change is respectively:

$$
\begin{aligned}
& \mathrm{D}_{\text {Area }}=\frac{\left|\operatorname{Area}\left(\mathrm{C}_{1}\right)-\operatorname{Area}\left(\mathrm{C}_{2}\right)\right|}{\max \left(\operatorname{Area}\left(\mathrm{C}_{1}\right), \operatorname{Area}\left(\mathrm{C}_{2}\right)\right)} \\
& \mathrm{D}_{\text {Level }}=\frac{\left|\mathrm{L}\left(\mathrm{C}_{1}\right)-\mathrm{L}\left(\mathrm{C}_{2}\right)\right|}{\max \left(\mathrm{L}\left(\mathrm{C}_{1}\right), \mathrm{L}\left(\mathrm{C}_{2}\right)\right)}
\end{aligned}
$$

Considering the difference of area and gray level, the noise can be avoided as an independent component, so the image segmentation can be realized.

$$
\operatorname{diff}\left(C_{1}, C_{2}\right)= \begin{cases}1 & \text { if } D_{\text {Level }} \geq T_{L} \text { and } D_{\text {Area }}<T_{A} \\ 0 & \text { elses }\end{cases}
$$

Among them, "1" indicates the existence of boundary, and " 0 " indicates that there is no boundary. TL and TA represent the gray change rate and the threshold of area change rate respectively. In the experiment, the segmented images are different, and the values of the two are also changed.

\section{This Algorithm Flow is Described in This Paper}

After the image component tree is set up, the algorithm description of the component merging based on the dual 
attributes of the gray and area is given.

(1) According to the input image component tree T, the area of each component in all grayscale levels is calculated.

(2) According to the merging criteria, the components are merged according to the order of gray level decreasing.

(3) According to the new component tree T ', output the segmented image $\mathrm{f}$.

\section{ANALYSIS OF EXPERIMENTAL RESULTS}

In this paper, two groups of experiments were designed. The first group was divided by a patient's liver CT (this patient has Liver pseudotumor), and the second group was divided by a Budd Chiari syndrome patient's liver CT. The value range of the selected TA is $(0.15 \sim 0.25)$, and the range of TL is $(0.50 \sim 0.60)$. The segmentation method of this paper is compared with the fuzzy clustering algorithm [8] and the Canny operator [9].

TABLE I THIS ALGORITHM AND OTHER RUNNING TIME COMPARISON UNIT: S

\begin{tabular}{|c|c|c|c|}
\hline Image & $\begin{array}{c}\text { Fuzzy } \\
\text { clustering } \\
\text { algorithm }\end{array}$ & $\begin{array}{c}\text { Canny } \\
\text { operator }\end{array}$ & $\begin{array}{c}\text { Algorithm in } \\
\text { this paper }\end{array}$ \\
\hline Figure 4 & 4.56 & 3.65 & 1.36 \\
\hline Figure 5 & 4.68 & 3.89 & 0.98 \\
\hline
\end{tabular}

(A)ORIGINAL

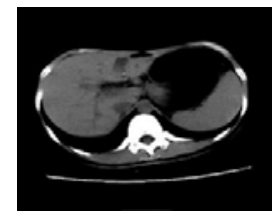

(B) LOG ENHANCEMENT

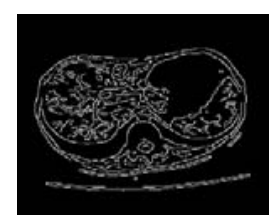

(C) FUZZY CLUSTERING ALGORITHM

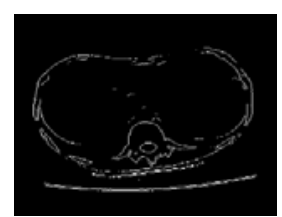

(D) CANNY OPERATOR

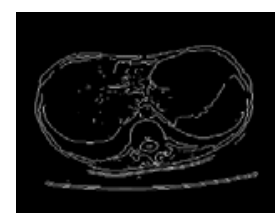

(E) ALGORITHM IN THIS PAPER

FIGURE IV. COMPARISON OF FIRST GROUP EXPERIMENTAL RESULTS

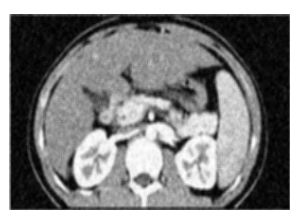

(A)ORIGINAL

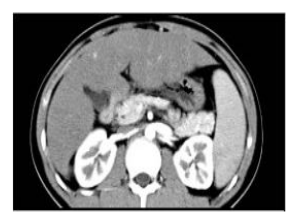

(B)LOG ENHANCEMENT

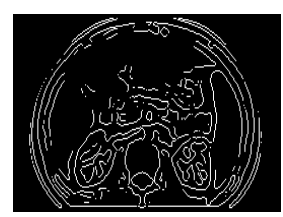

(C)FUZZY CLUSTERING ALGORITHM

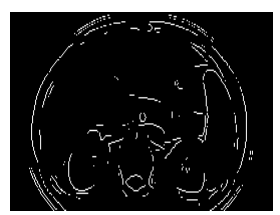

(D)CANNY OPERATOR

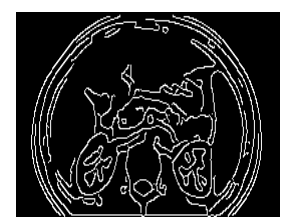

(E)ALGORITHM IN THIS PAPER

FIGURE V. COMPARISON OF SECOND GROUP EXPERIMENTAL RESULTS

In conclusion, compared with fuzzy $\mathrm{C}$ mean algorithm and Canny operator, it can be seen from segmentation results that the algorithm achieves ideal segmentation results. The running time of the algorithm is shorter.

\section{CONCLUSION}

In this paper, we study the CT image segmentation algorithm based on the similarity judgment of the adjacent component's regional attributes. Aiming at the uneven and fuzzy features of the image itself, the LOG is enhanced in the 
image preprocessing stage, so that the details of the image are enhanced while reducing the noise. Then, we use the immersion algorithm to build the component tree, and calculate the grayscale and area attribute data of tree nodes. According to the algorithm of adjacent component attribute similarity determination proposed in this paper, the nodes are specified. Finally, the purpose of image segmentation is achieved. The experimental results show that the algorithm proposed in this paper can achieve accurate image segmentation and the efficiency of the algorithm is higher. In this paper, the complex node attributes are designed in order to extend the image segmentation in the future.

\section{ACKNOWLEDGEMENT}

This research was financially supported by the National Science Foundation (No. 61572142).

\section{REFERENCE}

[1] P Chinmayi, L Agilandeeswari, M Prabukumar.Survey of Image Processing Techniques in Medical Image Analysis: Challenges and Methodologies, International Conference on Soft Computing \& Pattern Recognition, 2016:460-471.

[2] Guiping Jiang, Wenjian Qin, Shoujun Zhou, Changmiao Wang, State-ofthe-art in Medical Image Segmentation, Chinese journal of computer,38(6),1222-1242,2015.

[3] Aiju Li, Yujie Lie, Tingmei Wang, Wenliang. Medical Image Segmentation Based on Maximum Entropy Multi-threshold Segmentation Optimized by Improved Cuckoo Search Algorithm, $20158^{\text {th }}$ International Congress on Image and Signal Processing (CISP 2015), 2015: 470-475.

[4] Frank R J,Grabowski T J, Damasio H. Voxelvise percentagetissue segmentation of human brain Magnetic resonance images Proceedings of the 25th annual Meeting, Society for Neuro-science, Society for Neuroscience. Washington, DC, USA, 1995:694.

[5] Wang H, Suh J W, Das S, et al. Hippocampus segmentation using a stable maximum likelihood classifier ensemble algorithm. IEEE International Symposium on Biomedical Imaging: From Nano to Macro. Rotterdam, Holland, 2011: 2036-2040.

[6] Yuwei Wu, Jia Liang, Yuanquan Wang, A method for segmentation of the cardiac MR images based on GGVF Snake[J].Journal of image and Graphics, 2010,15(4):598-606.

[7] Edwin Carlinet. A compparetive review of component tree computation algorithms, IEEE Trans. on Image Processing, 2014, 23(9): 3885-3895.

[8] Sharma N, Ray A K, Sharma S, et al.Segmentation of medical images using simulated anneaking based fuzzy C Means algorithm. Interntional Journal of Biomedical Engineering Technology, 2009, 2(3): 260-278.

[9] Canny J, A computational approach to edge detection. IEEE Transactions on Pattern Analysis and Machine Intelligence, 1986, 8(6):679-698. 